\title{
SEARCH FOR ACTINIDES BY SECONDARY REACTIONS IN TUNGSTEN AND GOLD
}

\author{
E. ROSS. K. BÄCHMANN, K. H. LIESER. Z. MALEK.* D. MOLZAHN† and J. RUDOLPH
}

\begin{abstract}
Tungsten and gold have been irradiated with $24 \mathrm{GeV}$ and $10.8 \mathrm{GeV}$ protons, resp., and the possible production of actinide nuclides by secondary reactions has been investigated. The actinide fractions were chemically separated and $\alpha$-spectra taken. No $\alpha$-peaks which could definitely be attributed to actinide nuclides could be found. By taking different formation mechanisms into consideration. upper limits for the cross-sections were calculated and compared with the values expected.
\end{abstract}

\section{INTRODUCTION}

THE ANNOUNCEMENT of the possible identification of element 112 from Marinov et al.[1] has stimulated a number of investigations[2-10] dealing with secondary reactions induced by high energy protons. These studies have been carried out with the simultaneous purpose that secondary reactions might offer a possibility to produce superheavy elements before new heavy ion accelerators come into operation.

For the production of elements with an atomic number greater than that of the target element two mechanisms can be formulated: (a) High energy protons. which are scattered elastically at a target nucleus, are transferring enough energy to this nucleus to exceed the Coulomb barrier of a second target nucleus[1]. (b) The interaction of high energy protons with complex nuclei leads to fragments with enough energy to exceed the Coulomb barrier of the target nucleus[1].

Compared with a heavy ion accelerator secondary reactions have the following advantages: (i) The compound nuclei formed by fragments of the target nuclei and another target nucleus may be more neutron-rich than in reactions using a heavy ion accelerator because part of the fragments is neutron-rich and very shortlived. Neutron-rich compound nuclei, however, are closer to the $\beta$-stability line. In a heavy ion accelerator such neutron rich and short-lived nuclides cannot be used. (ii) In secondary reactions a large bulk of target material can be used and there are no problems of heating up the target. On the other hand the rate of forma- tion of desired products per unit volume is much lower in the case of secondary reactions.

Most of the previous investigations have been carried out by separating certain elements (under the assumption of an analogous chemical behaviour of the superheavy homologues) and by looking for spontaneous fission events. With the exception of the experiments of Marinov et al. $[1,2]$ these experiments turned out to be negative. Build-up products, however, could be identified using $C u$ as target $[7,11]$ and using $U$ as target $[3,5$, $6]$.

In the latter case only products could be found, which had atomic numbers between 94 and 97 . These investigations showed that the cross sections decrease very rapidly with increasing atomic number, so that the production of superheavy elements seems to be hopeless.

In the present study we have looked for actinides which might have been produced in $\mathrm{W}$ or $\mathrm{Au}$, resp. The determination of cross sections for the build-up of actinides from $\mathrm{W}$ or $\mathrm{Au}$, resp., would allow an exrrapolation to the upper limit of cross sections for superheavy nuclides to be expected from the build-up of U. Furthermore, a search for actinides has the advantage that they cannot be produced by spallation reactions from impurities. Marinov et al. [1] report that "actinides seem to be produced in W". Unik et al.[6] report: "No actinides were observed in the tungsten targets which could be definitely attributed to secondary reactions." Therefore till present no definite proof for the formation of actinide nuclides from $\mathrm{W}$ - or $\mathrm{Au}$-targets has been given.

\section{EXPERIMENTAL}

The irradiation data for the 3 targets which had been used are summarized in Table 1 . Unfortunately no unirradiated 
Table 1. Irradiation data of the target

\begin{tabular}{lccccc}
\hline Target & Weight [g] & $\begin{array}{c}\text { Irradiation } \\
\text { time [d] }\end{array}$ & $\begin{array}{c}\text { Number of } \\
\text { protons }\end{array}$ & Decay time & $\begin{array}{c}\text { Proton } \\
\text { energy } \\
{[\mathrm{GeV}]}\end{array}$ \\
\hline W I & 3.42 & 15 & $1.7 \times 10^{17}$ & $120 \mathrm{~d}$ & 24 \\
W II & 5.40 & 15 & $1.7 \times 10^{17}$ & $1.4 \mathrm{a}$ & 24 \\
Au I* & 14.1 & 60 & $2 \times 10^{17}$ & $5 \mathrm{a}$ & 10.8 \\
\hline
\end{tabular}

*Alloyed with $\approx 10$ per cent platinum.

samples of the target materials were available, so that no reliable analysis of trace impurities could be carried out.

From $\mathrm{W} I$ the following fractions have been separated: Au, $\mathrm{Hg}, \mathrm{Pt}, \mathrm{Os}, \mathrm{Bi}$ and the actinides. From W II and $\mathrm{Au} I$ only the actinide fraction has been separated. The chemical procedure was as follows:

\section{W I-target}

The cylindrical rod of tungsten metal was dissolved anodically (using a.c. to avoid passivation) in $2 n \mathrm{NaOH}$. The dissolution was carried out in the presence of carriers of Re, $\mathrm{U}, \mathrm{Ag}, \mathrm{Pt}, \mathrm{Hg}, \mathrm{Tl}, \mathrm{Au}, \mathrm{Os}, \mathrm{La}, \mathrm{Zr}, \mathrm{Pd}, \mathrm{Se}, \mathrm{Mo}, \mathrm{V}$, In and $\mathrm{Sb}$. Hydrazine and $\mathrm{MnSO}_{4}$ were added and the hydroxides precipitated and redissolved. The fraction of the actinides and lanthanides was purified by several cycles of fluoride and hydroxide precipitations.

The osmium was obtained by distillation during the dissolution of the fluoride in $\mathrm{H}_{3} \mathrm{BO}_{3}$ and conc. $\mathrm{HNO}_{3}$. The volatile products were absorbed in thiourea and $\mathrm{HCl}$.

The solution which remained after the distillation was concentrated and $\mathrm{Au}$ was extracted from $6 n \mathrm{HCl}$ with MIBK (methylisobutylketone). The Au-fraction was stripped with in $\mathrm{HCl}$ and reduced with hydroquinone. The elemental gold was centrifuged.

In the aqueous phase of the MIBK-extraction $\mathrm{Hg}$ was reduced with $\mathrm{SnCl}_{2}$ and precipitated as $\mathrm{Hg}_{2} \mathrm{Cl}_{2}$. From the remaining solution $\mathrm{Pt}$, Ir and $\mathrm{Th}$ were extracted with amylacetate. Pt was obtained by precipitation as PtS.

After adding Bi-carrier to the aqueous phase $\mathrm{Bi}$ was precipitated with $\mathrm{NaOH}$ as $\mathrm{Bi}(\mathrm{OH})_{3}$. The hydroxide was dissolved and $\mathrm{BiHPO}_{4}$ precipitated with $\mathrm{Na}_{2} \mathrm{HPO}_{4}$. For the separation of $\mathrm{Hf}$ the precipitation was dissolved in $\mathrm{HCl}$ and extracted with trioctylamine.

\section{W II-target}

The W II sample was dissolved anodically as described above. For the separation from the bulk of $\mathrm{WO}_{4}^{-}$the solution was passed through a cation exchange (Dowex 50WX4) column, the $\mathrm{WO}_{4}^{--}$was eluated with $\mathrm{NaOH}$, and the Ac$\mathrm{La}-$ fraction was eluated with conc. $\mathrm{HCl}$ containing Lacarrier. Ba- and $\mathrm{Zr}$-carrier were added and the hydroxides precipitated. Several cycles of hydroxide and fluoride precipitations were carried out for purification. Then the solution was given on a cation exchange column and eluated with a solution of 20 per cent ethanol and 80 per cent conc. $\mathrm{HCl}$. The first $20 \mathrm{ml}$ of the eluate contained the trivalent actinides and the heavy lanthanides. After adding $0.05 \mathrm{mg} \mathrm{La}$-carrier the hydroxides were precipitated, dissolved and separated on another cation exchange column by eluation with $0.5 \mathrm{~m}$ $\alpha$-hydroxiisobutyric-acid (pH 4). The fraction containing the trivalent actinides with the exception of Ac was taken from the eluate. This fraction was evaporated on a fold foil and organic substances destroyed by heating up to $500^{\circ} \mathrm{C}$. The sample was suitable for $\alpha$-spectroscopy.

The yield of the separation had been measured by carrying out several separations with tracer nuclides under the same conditions. The yields were always between 60 and 70 per cent.

\section{Au I}

The separation of the actinide fraction was carried out as described for the W II-target with some minor changes. For the gold sample Am-241 was added to determine the yield which was found to be 62 per cent. To avoid contamination by the Am-241, an activity of only about $1 \mathrm{dph}$ was added. The purity of the added Am-241 was checked by $\alpha$-spectroscopy.

\section{RESULTS AND DISCUSSION}

\section{Spontaneous fission}

The fractions which had been separated from the W I-sample were measured for spontaneous fission events using proportional counters. The calibration was carried out with $\mathrm{Cf}-252$. The background of these detectors was in the range of 1 event in 3-6d. All spontaneous fission events measured were in between the statistical errors of the background. Since, however, only $3.4 \mathrm{~g} \mathrm{~W}$ with an integral flux of $2 \times 10^{17}$ protons has been used this result is not in contradiction to the result of Marinov et al. $[1,2]$. This group has irradiated $30 \mathrm{~g} \mathrm{~W}$ with an integral flux of about $10^{18}$ protons and obtained about 1 fission per day.

\section{Discussion of the $\alpha$-spectra}

From the actinide fraction of the sample W I only one measurement of the $\alpha$-activity was carried out without any identification (104 ipm with about 50 per cent detector efficiency). From the samples W II and Au I the $\alpha$-spectra (Figs. 1 and 2) were taken as a function of time. The yield of the detector was 10.1 per cent. In the W II-sample the following nuclides were identified unambiguously: Po-210, Ac-227 and its daughter products Th-227, Ra-223, Rn-219, Po-215, Bi-211 and Po-211.

A quantitative investigation led to the following results: (a) Comparing the activity of Th-227 and $\mathrm{Ra}-223$ it is found that the activity of Ra-223 and its daughter products is too high compared with equilibrium. (b) The decay curves of the peaks attributed to Ra-223 and the daughter products followed initially the decay of Ra-223. 


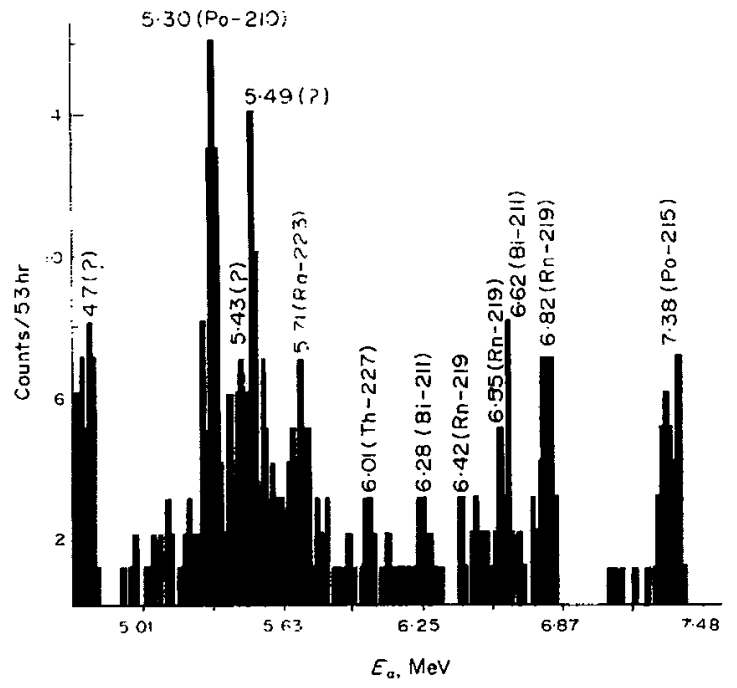

Fig. 1. Alpha-spectrum of the W II-sample. Energy calibration has been carried out with standards of Pu-239 (5.16 MeV). Am-241 (5.49 MeV) and Cm-244 (5.81 MeV).

After some months, however, nearly constant activities were observed.

These results can be explained by assuming a good separation of Th from the sample but a poorer one of $\mathrm{Ra}$ and Ac, resp. Result (a) can thus be explained by decay of the Ra-223 still present, result (b) can be interpreted by the presence of small amounts of Ac- 227 .

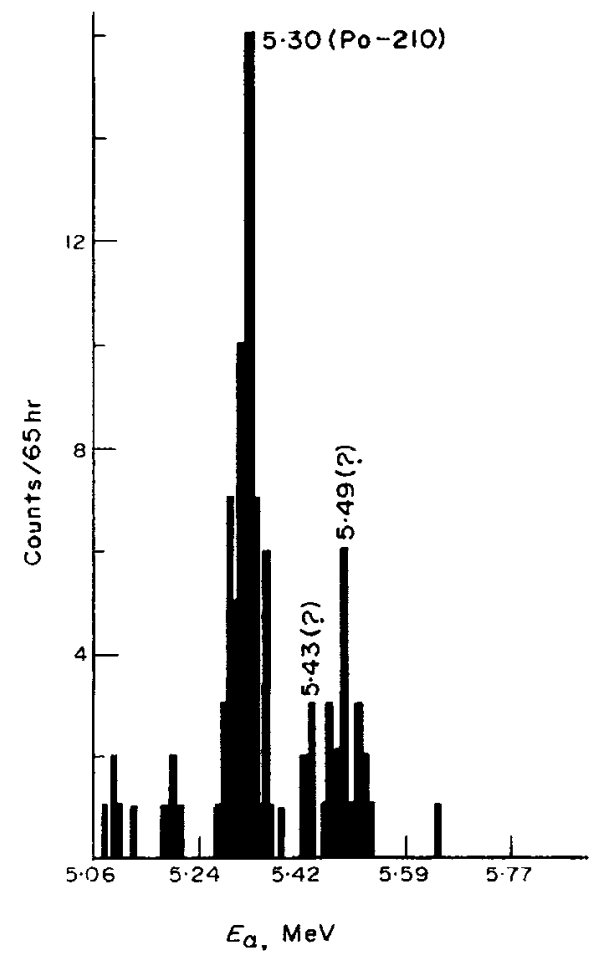

Fig. 2. Alpha-spectrum of the Au I-sample. Energy calibration as described in Fig. 1.
It was not possible to explain the broad peak at 5.45 $\mathrm{MeV}$ and the peak at $4.7 \mathrm{MeV}$ in the W II-spectrum. The $5.49 \mathrm{MeV}$ line cannot be attributed to Th-228 and its daughter products since the $8.78 \mathrm{MeV}$ line of $\mathrm{Po}-212$ is missing. The long-lived $4.7 \mathrm{MeV}$ line might be attributed to Th-230 or Bi-210. The half life of Th-230, however, is too long and no detectable activity should be present. $\mathrm{Bi}-210$ is short-lived, but it may be present as daughter product of $\mathrm{Pb}-210$, but $\mathrm{Pb}-210$ produces Po-210 in radioactive equilibrium. The peak of Po-210, however, decayed with a half-life of $140 \mathrm{~d}$ which means that $\mathrm{Pb}-210$ and therefore $\mathrm{Bi}-210$ cannot be present. In the Au I sample the most prominent peak is the peak of Po-210. The other peaks decayed with a half-life of about 10 days. They can all be attributed to $\mathrm{Ra}-223$ and its daughter products, but two peaks at 4.74 and $5 \cdot 20$ cannot be identified.

For the nuclides which can be identified the following formation mechanisms can be discussed: 1. Spallation of U-238, U-235 or Th-232, which could have existed as ppm impurities in the $\mathrm{W}$ - or Au-sample. 2. Decay of $\mathrm{U}-238, \mathrm{U}-235$ or $\mathrm{Th}-232$, which could have existed as ppm impurities in the W- or Au-sample. 3. Formation by build-up reactions from $\mathrm{W}$ or Au.

$\operatorname{Ad}(1)$

Assuming $1 \mathrm{ppm}$ impurity of $U$ in the samples the activities which have been found could have been easily produced by spallation of U. An exact calculation cannot be carried out, since the chemical yields of Ac-227. Ra-223 and Po-210 could not be measured. Th-228 and Th-227, however, should have been produced by spallation with about the same cross sections. Since thorium has been separated, Ra-224 and the daughter products from Th- 228 should be in the $\alpha$-spectra. Since they have not been found although the time between separation and measurement has been short enough. we think that spallation can be excluded.

$\operatorname{Ad}(2)$

The lines in the $\alpha$-spectra can be attributed to the decay products of U-235 which could have been present as an impurity in the W- or Au-sample. The absence of lines of daughter products of U-238 is to be understood as being due to the half-life of Th-230 being too long. A comparison of the daughter products of U-235 and $\mathrm{U}-238$ is difficult since it is not known how the radioactive equilibria have been established after the production of the tungsten metal or the gold metal, resp.

Ad (3)

Principally the formation of $\mathrm{Ac}-227$ or $\mathrm{Th}-227$ by build-up reaction cannot be excluded. Yet the absence of Th-228 and daughter products is in contradiction to the assumption of a build-up reaction.

The formation of Po-210 could also be explained by a build-up reaction. In this case one should, however. also find Po-208 even with higher yields becaulse Po-208 is 
more neutron deficient. With this argument a $\operatorname{Bi}(p, \gamma)$ Po-210-reaction can be excluded too, since a $(p, 2 n)$ reaction from $\mathrm{Bi}$ to Po-208 should be much more probable. The presence of Po-210 (precursor U-238) is to be explained as a daughter product of $\mathrm{Pb}-210$ which remained during the production of the metals. Since the chemical yield of Po has not been determined it is not possible to say whether the higher peak of $\mathrm{Po}-210$ in the Au-spectrum is due to a higher content or due to a higher yield because of the slightly modified separation.

Of much greater interest was the formation of nuclides with an atomic number equal to or higher than 94. These nuclides should have been formed by a buildup reaction, since the formation from impurities by decay or spallation reaction is not possible. Due to the chemical separation which has been carried out a certain amount of the $\mathrm{Pu}$ could have been still in the samples but with an unknown yield. Due to the irradiation time and the decay time (Table 1) of our study only nuclides are of interest with half-lives between $150 \mathrm{~d}$ and 30 a, such as $\mathrm{Cm}-242, \mathrm{Cf}-248, \mathrm{Cm}-244$, $\mathrm{Pu}-236$, Pu-237, $\mathrm{Cm}-241$ and their daughter products. In Table 2 these nuclides are summarized, as well as the half-lives and the $\alpha$-energies. Nuclides which are too neutron excessive $(N / Z>1.54)$ have not been taken into account.

The upper limits of the disintegration rates which have been taken from the $\alpha$-spectra are also summarized in Table 2. Decay, chemical yield and detector efficiency have been taken into account for this calculation. The values for the upper limit in Table 2 have large uncertainties, especially if a peak of another nuclide is near by. For example Cf-248 should show a peak at 6.26 MeV. Part of this peak is to be attributed to the peak of Bi-211 at $6.28 \mathrm{MeV}$. If the ratio of the peak at $6.62 \mathrm{MeV}(\mathrm{Bi}-211)$ and $6.28 \mathrm{MeV}$ is taken then the peak at 6.26 seems to be too high. On the other hand a decay analysis of the peak at 6.26 results in a half-life too long compared with the 6.62 peak. But any estimate of a possible contribution of $\mathrm{Cf}-248$ is connected with large errors.

In the Au-sample no peaks could be found after 6 months (with the exception of Po-210). Therefore the minimum detectable $\alpha$-rate which could be measured has been taken as upper limit.

A further possible identification of the actinide nuclides would have been a measurement of the X-rays. However, it was impossible to identify any line of the actinides in the $X$-ray spectrum, since the remaining rare earth nuclides give a large background of low energy gammas.

\section{Production of actinide nuclides}

The maximum $\alpha$-activities which are summarized in Table 2 can be used to estimate the maximum crosssections for the formation of these nuclides.

The formation of a nuclide with an atomic number $Z$ which is higher than that of the target, as for instance the formation of an actinide in tungsten or gold, may be described by the following equation:

$$
\frac{\mathrm{d} N_{3}}{\mathrm{~d} t}=\varphi_{2} \cdot f_{\mathrm{C}} \sigma_{2} \cdot N_{1}^{\prime}
$$

where $\varphi_{2}$ is the flux of any kind of heavy ions, $f_{c}$ the fraction of these ions having energies greater than the coulomb barrier, $\sigma_{2}$ the cross-section for the production of a certain actinide and $N_{1}^{\prime}$ the number of target nuclei which are hit by the flux $\varphi_{2}$.

The production rate of heavy ions by the incident protons is given by the equation

$$
\frac{\mathrm{d} N_{2}}{\mathrm{~d} t}=\varphi_{1} \cdot \sigma_{1} \cdot N_{1}
$$

where $\varphi_{1}$ is the flux of the protons, $\sigma_{1}$ the cross-section for the formation of any kind of heavy ions by any mechanism and $N_{1}$ the number of atoms in the target. From Eqn (2) it follows

$$
\varphi_{2}=\frac{\mathrm{d} N_{2}}{\mathrm{~d} t} \cdot \frac{1}{q}
$$

where $q$ is the area of the target perpendicular to the beam in $\mathrm{cm}^{2}$.

Whereas the absorption of high energy protons in the target is negligible, the range of the heavy ions having an energy higher than the coulomb barrier $E_{C}$ which are produced by the proton beam is very short. In order to take this into account the following equation is applied:

$$
N_{1}^{\prime}=N_{1} \frac{R}{d}
$$

where $R$ is the mean range of the heavy ions with energies $E>E_{C}$ and $d$ the thickness of the target (both in $\mathrm{cm}$ ). This relation is only valid if $R \ll d$.

Table 2. Upper limits for the disintegration rates of actinides nuclides

\begin{tabular}{lcccc}
$\begin{array}{c}\text { Actinide } \\
\text { nuclide }\end{array}$ & Half life[12] & $\alpha$-energy (MeV)[12] & $\begin{array}{c}\text { Maximum disintegration } \\
\text { rate (corrected) W II }\end{array}$ & $\begin{array}{c}\text { Maximum disintegration } \\
\text { rate (corrected) Au I }\end{array}$ \\
\hline Pu-236 & $2.85 \mathrm{a}$ & $5 \cdot 77 ; 5.72$ & $10 \times 10^{-4} \mathrm{dps}$ & $1 \times 10^{-4} \mathrm{dps}$ \\
$\mathrm{Cm}-241$ & $35 \mathrm{~d}$ & $5.94 ; 5.93$ & $100 \times 10^{-4} \mathrm{dps}$ & $1 \times 10^{-4} \mathrm{dps}$ \\
$\mathrm{Cm}-242$ & $163 \mathrm{~d}$ & $6.12 ; 6.08$ & $8 \times 10^{-4} \mathrm{dps}$ & $-\mathrm{d}$ \\
$\mathrm{Cm}-243$ & $32 \mathrm{a}$ & $5 \cdot 79 ; 5.741$ & $8 \times 10^{-4} \mathrm{dps}$ & $1 \times 10^{-4} \mathrm{dps}$ \\
$\mathrm{Cm}-244$ & $18 \mathrm{a}$ & $5.81 ; 5.77$ & $8 \times 10^{-4} \mathrm{dps}$ & $1 \times 10^{-4} \mathrm{dps}$ \\
$\mathrm{Cf}-248$ & $350 \mathrm{~d}$ & $6.26 ; 6.22$ & $5 \times 10^{-4} \mathrm{dps}$ & $1 \times 10^{-4} \mathrm{dps}$ \\
\hline
\end{tabular}

* $\alpha$-Branching of 1 per cent. 
With Eqns (2)- (4). Eqn (1) becomes

$$
\frac{\mathrm{d} N_{3}}{\mathrm{~d} t}=\varphi_{1} \cdot N_{1}^{2} \cdot \sigma_{1} \cdot \sigma_{2} \cdot f_{C} \cdot \frac{R}{q \cdot d} .
$$

By integration the activity of a certain actinide is obtained

$$
A_{3}(t)=\varphi_{1} \cdot N_{1}^{2} \cdot \sigma_{1} \cdot \sigma_{2} \cdot f_{\mathrm{C}} \cdot \frac{R}{q \cdot d}\left(1-e^{-\lambda_{3} t}\right)
$$

where $i_{3}$ is the decay constant of this nuclide.

By substituting in Eqn (6)

$$
\sigma_{0}=\sigma_{1} \cdot f_{C} \cdot \sigma_{2} \cdot \frac{R \cdot N_{1}}{q \cdot d}
$$

the following relation is obtained

$$
A_{3}(t)=\rho_{1} \cdot \sigma_{0} \cdot N_{1}\left(1-e^{-i_{3} t}\right) .
$$

Since for the target

$$
N_{1}=\frac{m}{M} \cdot N_{L}
$$

where $m$ is the mass, $M$ the atomic mass of the target and $N_{L}$ Avogadro's number, one may write instead of (7)

$$
\begin{aligned}
\sigma_{0} & =\sigma_{1} \cdot f_{C} \cdot \sigma_{2} \cdot \frac{\rho \cdot N_{L}}{M} \cdot R \\
& =\sigma_{1} \cdot f_{C} \cdot \sigma_{2} \cdot R^{\prime} .
\end{aligned}
$$

In these equations the formation of a certain nuclide of higher atomic number $Z$ by different reactions with heavy ions is taken into account. Besides the direct formation this nuclide may also be produced by decay of another nuclide.

The most interesting values for the future when heavy ion accelerators will be in use, are the $\sigma_{2}$ values. For tungsten and gold $\frac{\rho \cdot N_{L}}{M}=6 \cdot 31 \cdot 10^{22}$ and $5 \cdot 91 \cdot 10^{22}$ $\mathrm{cm}^{-3}$, respectively.

For the formation of nuclides heavier than the target nuclide the following three reaction mechanisms can be discussed: (a) Fusion-fission (formation of compound nucleus followed by fission). (b) Fusion-evaporation (compound nucleus formation followed by neutron evaporation). (c) Transfer-reactions.

In Fig. 3 part of the nuclide chart in the region of the actinides is shown. In this figure, two lines are drawn. the FF (fusion-fission)-line, and the FE (fusionevaporation)-line representing the 1 per cent probability limit for the formation of nuclides by the FFmechanism and the FE-mechanism, resp. Nuclides which are on the right of these lines are very unlikely to be produced by one of these mechanisms. The FF. line has been calculated with the following assumptions: Formation of a compound nucleus and symmetric fission with an unchanged charge distribution (UCDrule) and no evaporation of neutrons. This is a rather unrealistic assumption and will probably occur for less than 1 per cent.

For the calculation of the FE-line the following assumptions have been made: Formation of fragments with a neutron-to-proton ratio of about 1.40 and evaporation of about 4 neutrons ( $(\mathrm{HI}, ;)$-reactions are very unprobable[13]). Trabitzsch and Bächmann[14] have found that the cross-sections of Rb-isotopes with $N / Z$-rations $=1.40$ are only 1 per cent of the maximum cross-sections.

For mechanism (c) (transfer reactions) no line can be drawn. In principle it is possible to formulate all kinds of transfer reactions which lead even to very neutron rich nuclides.

In Table 3 these three mechanisms are compared for the example of the formation of Cm-242 from $W$ and $U$. In the latter case Cm-242 is very likely to be formed by a reaction with a fragment and subsequent evaporation of neutrons. For U-238 as target the value for the overall cross-section given by Westgaard et al.[3] has been taken. Using Eqn (8) the $\sigma_{0}$-values are calculated by inserting the maximum disintegration rates of Table 2 . For the compound nucleus formation and the transfer reaction $\mathrm{Ti}-60$ and $\mathrm{Kr}-86$, resp., have been assumed as fragments. These nuclides are neutron rich. $\mathrm{Kr}-86$ is

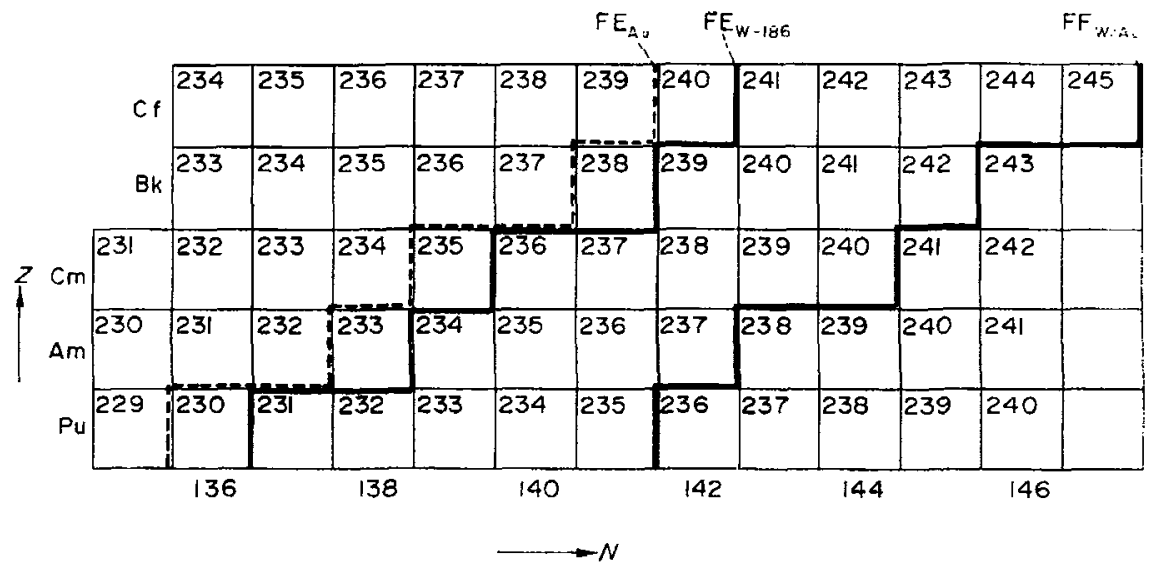

Fig. 3. N, Z-plot of the calculated 1 per cent probability line for the production of actinide nuclides by different build-up mechanisms. For further explanation. see text. 
Table 3. Formation of $\mathrm{Cm}-242$ by build-up reactions with $\mathrm{W}$ and $\mathrm{U}$

\begin{tabular}{|c|c|c|c|c|}
\hline \multirow[b]{2}{*}{ Mechanism } & \multicolumn{3}{|c|}{ W-186 } & \multirow{2}{*}{$\begin{array}{c}\text { U-238 } \\
\text { Compound nucleus } \\
\text { evaporation of } \\
\text { neutrons }\end{array}$} \\
\hline & neutrons & Fusion-fission & Transfer & \\
\hline Reaction & ${ }^{186} \mathrm{~W}\left({ }^{60} \mathrm{Ti}, 4 n\right)^{242} \mathrm{Cm}$ & ${ }^{186} \mathrm{~W}\left({ }^{186} \mathrm{~W} ;{ }^{126} \mathrm{Te}, 4 n\right)^{242} \mathrm{Cm}$ & ${ }^{184} \mathrm{~W}\left({ }^{86} \mathrm{Kr},{ }^{28} \mathrm{Si}\right){ }^{242} \mathrm{Cm}$ & ${ }^{238} \mathrm{U}\left({ }^{8} \mathrm{Be}, 4 n\right)^{242} \mathrm{Cm}$ \\
\hline Product & $\mathrm{Cm}-242$ & $\mathrm{Cm}-242$ & $\mathrm{Cm}-242$ & $\mathrm{Cm}-242$ \\
\hline$N$ & $1.1 \times 10^{22}$ & $1.1 \times 10^{22}$ & $1.1 \times 10^{22}$ & - \\
\hline$\varphi\left(\mathrm{cm}^{-2} \sec ^{-1}\right)$ & $2 \times 10^{11}$ & $2 \times 10^{11}$ & $2 \times 10^{11}$ & - \\
\hline$\sigma_{0(\exp )}($ barn $)$ & $5.5 \times 10^{-21}$ & $5.5 \times 10^{-12}$ & $5.5 \times 10^{-12}$ & $3 \times 10^{-11}[3]$ \\
\hline$\sigma_{1}$ & $10^{-5}$ mbarn & 6 nharn[7] & $0.01 \mathrm{mbarn}[14]$ & 15 mbarn $[21]$ \\
\hline$f_{c}$ & $10^{-2}$ & $0 \mathrm{nbarn}[\mathrm{I}]$ & $10^{-4}$ & $0 \cdot 1$ \\
\hline$R\left(\mathrm{mg} / \mathrm{cm}^{2}\right)$ & 3 & 0.5 & 1.5 & 50 \\
\hline$R^{\prime}\left(\right.$ atoms $\left./ \mathrm{cm}^{2}\right)$ & $9.8 \times 10^{18}$ & $1.6 \times 10^{18}$ & $4.9 \times 10^{18}$ & $127 \times 10^{18}$ \\
\hline$\sigma_{2}($ barn) & $10^{-4}$ & $10^{-5}$ & $10^{-6}$ & $10^{-4}$ \\
\hline$\sigma_{0 \text { (est,) }}($ barn) & $1.15 \times 10^{-19}$ & $1.2 \times 10^{-19}$ & $5 \times 10^{-21}$ & $2 \times 10^{-11}$ \\
\hline
\end{tabular}

formed with a reasonable independent cross-section which can be estimated quite well[14]. Ti-60 is very neutron rich so that only a rough estimation of the cross-section can be given.

Much more difficult is the estimation of the contribution of fragments having energies exceeding the Coulomb barrier. Direct counter techniques of Poskanzer, Butler and Hyde[21] lead to the result that part of the fragments with lower $Z$ exceeds the Coulomb barrier. For example Ar shows tailing to higher energies. Differential kinetic energy measurements for large fragments have been carried out only by a few authors[1517]. A study of the differential ranges of $\mathrm{Cu}-64$ produced by the interaction of $2.2 \mathrm{GeV}$ protons with uranium[15] showed no fragments with energies higher than 140 $\mathrm{MeV}$. Using the background of the measurements as the upper limit one can estimate that less than $10^{-3}$ of the $\mathrm{Cu}$-fragments have energies higher than $140 \mathrm{MeV}$. Therefore a value of $10^{-2}$ for Ti- 60 and a value of $10^{-4}$ for $\mathrm{Kr}-86$ has been taken. The factor $R^{\prime}$ is estimated by the range of the fragments[18] (Ti-56, W-186, Kr-86) and the excitation function (FWHM as energy window).

For the $\sigma_{2}$-value of the FE-mechanism a value of $10^{-4} \mathrm{~b}$ has been taken. This is a value which has been measured, e.g. in the reaction ${ }^{232} \mathrm{Th}\left({ }^{13} \mathrm{C}, 5 n\right)^{240} \mathrm{Cm}$ : 0.7 mbarn[19] or ${ }^{238} \mathrm{U}\left({ }^{12} \mathrm{C}, 4 n\right)^{246} \mathrm{Cr}: 30 \mu$ barn[20]. This value is to be considered as a maximum value since the ratio $\Gamma_{n} / \Gamma_{f}$ depends on the angular momentum which has been transferred. In the reaction ${ }^{186} \mathrm{~W}\left({ }^{60} \mathrm{Ti}, 4 n\right){ }^{242} \mathrm{Cm}$ more rotational energy is transferred. In the region of the actinides $\Gamma_{n} / \Gamma_{f}$ does not depend, at high excitation energies, on the deposition energy[22].

For the FF-mechanism a $\sigma_{2}$ of $10^{-5}$ barn was assumed. This low yield has been taken because the fission to $\mathrm{Cm}-242$ is quite asymmetric and $\mathrm{Cm}-242$ is not in the maximum of the charge dispersion (taking the UCD rule). The most uncertain value is the one for the transfer reaction.

Inserting the above estimated values in Eqn (10) it is found that all $\sigma_{0 \text { (est.) }}$ are much lower than the upper experimental values given by the background of the measurements.

The fact that we could not really show the existence of an actinide nuclide is a proof that we can exclude unknown effects causing a tremendous increase in the cross-sections.

One may ask whether it is possible to determine the highest estimated cross-section of $10^{-19}$ barn by $\alpha$ counting: A factor of $5 \times 10^{7}$ which is needed compared with our experiment is very difficult to obtain, e.g. an irradiation of $2 \mathrm{~kg} \mathrm{~W}$ with $6 \times 10^{18}$ protons and a counting time of 300 days would result in a factor of $3 \times 10^{6}$. It is not possible to exclude completely the possibility of the formation of actinides by secondary reactions since most of the estimates are quite crude especially for the transfer reaction.

The last important question is whether it is worth while to look for superheavy nuclides using secondary reactions. Marinov et al. $[1,2]$ have found $\sigma_{0} \approx$ $1.2 \times 10^{-16}$ barn for spontaneous fission in a $\mathrm{Hg}$. sample under the assumption that the source gives 1 fission per day and the half life is 1 month. This value would correspond to about 12 mbarn for $\sigma_{2}$ which is quite high a cross-section and only reasonable if one assumes that the fissionability of a superheavy nuclide is quite low. Comparing mechanism FF and FE, the fusion-fission-mechanism is more favourable because the FE-mechanism leads to a nucleus which is too neutron deficient. The fusion-fission-mechanism, however, leads only to the formation of a superheavy nucleus under the assumption of a very asymmetric fission.

$$
\begin{aligned}
{ }^{186} W+{ }^{186} W & \rightarrow{ }^{372} 148 \\
{ }^{372} 148 & \rightarrow{ }^{316} 126+{ }^{58} 22+4 n \\
{ }^{316} 126 & \rightarrow 112+x \alpha+x^{\prime} \beta .
\end{aligned}
$$

Recent calculated values for the half-lives of fission, $\alpha$-decay and $\beta$-decay of superheavy nuclei[23] show that a fusion of two tungsten nuclei followed by fission 
(UCD-rule) will only lead to long-lived nuclides, if the heavy fragment has $Z=126$. In the case of $Z=120$ or $Z=114$ the fission fragment will decay by $\alpha$-emission and miss the stable island.

Another chance would be to increase the overall cross-section by a combination of mechanism FF and FE. e.g. using a mixed target of $\mathrm{Ca}-48$ and $\mathrm{Pu}-242$ (as oxides) the following reaction could take place:

$$
{ }_{94}^{242} \mathrm{Pu}+{ }_{20}^{48} \mathrm{Ca} \rightarrow{ }_{114}^{286} 114+4 n \text {. }
$$

Now the $\sigma_{1}$ of Eqn (10) could be much higher and due to the application of a neutron rich target nucleus and projectile the compound nucleus would not be too neutron deficient. By elastic scattering $\mathrm{Ca}-48$ would much more easily obtain an energy sufficient to overcome the Coulomb barrier than W-186.

Acknowledgement-The authors wish to thank Prof. Dr. W. Gentner and Dr. O. Müller for the irradiated W and Au. They are grateful to Prof. Dr. R. Brandt for help in measuring the spontaneous fission events.

This research was performed by financial support of the Bundesministerium für Bildung und Wissenschaft.

\section{REFERENCES}

1. A Marinov, C. J. Batty, A. I. Kilvington, J. L. Weil, A. M. Friedman, G. W. A. Newton, V. J. Robinson, J. D. Hemingway and D. S. Mather, Nature, Lond. 234, 212 (1971).

2. A. Marinov, C. J. Batty, A. I. Kilvington, G. W. A. Newton, V. J. Robinson and J. D. Hemingway, Nature, Lond. 229, 464 (1971).

3. L. Westgaard, B. R. Erdal, P. G. Hansen, E. Kugler, G. Sletten, S. Sundell, T. Fritsch, E. Henrich, W. Theis, G. K. Wolf, J. Camplan, R. Klapisch, R. Meunier.
A. M. Poskanzer, C. Stephan and J. Tys, Nucl. Phr.. A192, 517 (1972).

4. R. Brandt, D. Molzahn and P. Patzelt. Radiochim. Acta.

5. R. A. Esterlund, A. F. Stehney and E. P. Steinberg. Nucl. Phys. A179, 645 (1972).

6. J. P. Unik, E. P. Horwitz. K. L. Wolf. I. Ahmad. S. Fried, D. Cohen, P. R. Fields. C. A. A. Bloomquist and D. J. Henderson, Nucl. Phys. Al91. 233 (1972).

7. S. Katcoff and M. L. Perlman, Nature, Lond. 231, 522 (1971).

8. G. N. Flerov, Yu. P. Gangrsky and O. A. Orlova, Dubna E 7-5887, 1971.

9. E. Ross, K. Bächmann and K. H. Lieser. Inorg. mucl. Chem. Lett. 8, 417 (1972).

10. Yu. A. Batusov et al., Report P1-6551: KFK-tr-424. 1972.

11. K. E. Dierenfeldt: Thesis, University of California. 1966.

12. J. C. Post, Actinides Ret:1, 55 (1967).

13. T. Sikkeland. Ark. Fysik 36, 539 (1966).

14. U. Trabitzsch and K. Bächmann, Radiochim. Acta 16. 15 (1971).

15. K. Bächmann and J. B. Cumming. Phrw. Ret. C5. 210 (1972).

16. V. P. Crespo, J. B. Cumming and A. M. Poskanzer Phys. Rev. 174, 1455 (1968).

17. J. B. Cumming, R. J. Cross, J. Hudis and A. M. Poskanzer, Phys. Rev. 134B, 167 (1964).

18. L. C. Northcliffe and R. F. Schilling. Nuclear Data Tables A7, 233 (1970).

19. G. N. Flerov, Second International Conference on the Peaceful Uses of Atomic Energy. Geneva, P/22/99. Vol. 14.

20. Cited in E. K. Hyde, I. Perlman and G. T. Seaborg. The Nuclear Properties of the Heaty Elements. Vol. 1. p. 350 .

21. A. M. Poskanzer, G. W. Butler and E. K. Hyde, Phys. Rev. C3, 882 (1971).

22. R. L. Hahn and H. W. Bertini, Phys. Rer. C6, 660(1972)

23. E. O. Fiset and J. R. Nix, Nucl. Phys. A139. 647 (1972). 